\title{
AN ACCRETION MODEL FOR THE GROWTH OF THE CENTRAL BLACK HOLE ASSOCIATED WITH IONIZATION INSTABILITY IN QUASARS
}

\author{
Y. Lu ${ }^{1}$ \\ National Astronomical Observatories, Chinese Academy of Sciences, Beijing 100012, China; ly@bao.ac.cn \\ K. S. CHENG \\ Department of Physics, University of Hong Kong, Hong Kong, China \\ AND \\ S. N. ZHANG ${ }^{2}$ \\ Physics Department and Center for Astrophysics, Tsinghua University, Beijing 100084, China \\ Received 2002 November 19; accepted 2003 February 26
}

\begin{abstract}
A possible accretion model associated with the ionization instability of quasar disks is proposed to address the growth of the central black hole $(\mathrm{BH})$ harbored in the host galaxy. The evolution of quasars in cosmic time is assumed to change from a highly active state to a quiescent state triggered by the S-shaped ionization instability of the quasar accretion disk. For a given external mass transfer rate $\dot{M}_{\text {ext }}$ supplied by the quasar host galaxy, ionization instability can modify the accretion rate in the disk and separate the accretion flows of the disk into three different phases, like an S-shape. We suggest that the bright quasars observed today are those quasars with disks in the upper branch of the S-shaped instability, and the faint or "dormant " quasars are simply these systems in the lower branch. The middle branch is the transition state, which is unstable. We assume the quasar disk evolves according to the advection-dominated inflow-outflow solution (ADIOS) configuration in the stable lower branch of the S-shaped instability, and the Eddington accretion rate is used to constrain the accretion rate in the highly active phase. The mass ratio between a $\mathrm{BH}$ and its host galactic bulge is a natural consequence of an ADIOS. Our model also demonstrates that a seed BH $\left(\sim 2 \times 10^{6} M_{\odot}\right)$ similar to those found in spiral galaxies today is needed to produce a BH with a final mass of $\sim 2 \times 10^{8} M_{\odot}$.

Subject headings: accretion, accretion disks — black hole physics — galaxies: active — galaxies: bulges — galaxies: nuclei — quasars: general
\end{abstract}

\section{INTRODUCTION}

Nuclear feeding is most important in the evolution of quasars and quasar host galaxies. Since quasars were first discovered, they have been suggested to be powered by the accretion of gas onto supermassive black holes (SMBHs) at the center of galaxies (Lynden-Bell 1969). In the framework of the hierarchical dark matter cosmology (Haehnelt, Natarajian, \& Rees 1998), the formation and evolution of galaxies and their active nuclei are intimately related (Sanders \& Mirabel 1996; Boyle \& Terlevich 1998; Fabian 1999; Granato et al. 2001; Haehnelt \& Kauffmann 2000). Consequently, the growth and the fueling of the SMBH and the construction of the galactic bulge are now seen as being potentially related processes (Silk \& Rees 1998; Fabian 1999; Granato et al. 2001). Submillimeter photometry of eight X-ray-absorbed active galactic nuclei (AGNs; Page et al. 2001) reveals that active nuclei and their galaxies evolve together, with the black hole $(\mathrm{BH})$ accreting matter and the galaxy creating stars. On the other hand, recent high-resolution observations of galactic centers have revealed that the estimated mass of the central "massive dark object," which is the nomenclature for an SMBH candidate, correlates with the mass of the galactic bulge. The $\mathrm{BH}$-bulge mass

\footnotetext{
${ }^{1}$ Visiting Scholar, Department of Physics, University of Hong Kong, Hong Kong, China.

2 Also at Physics Department, University of Alabama, Huntsville, AL 35899.
}

relation, with $M_{\mathrm{bh}} \sim(0.001-0.006) M_{\text {bulge }}$ (Kormendy \& Richstone 1995; Magorrian et al. 1998; van der Marel 1999; Gebhardt et al. 2000a; Ferrarese \& Merritt 2000) for normal galaxies, was shown to extend to nearby quasar host galaxies as well (Laor 1998; Nelson et al. 2000; Ferrarese et al. 2001). Based on the remarkable dependence of $\mathrm{BH}$ mass on spheroid velocity dispersion (Ferrarese \& Merritt 2000; Merritt \& Ferrarese 2001; Gebhardt et al. 2000a, 2000b), several theoretical models for $\mathrm{BH}$ growth have been considered to explain the $\mathrm{BH}$ to-bulge correlations, e.g., a hydrodynamic model, including wind regulation (Silk \& Rees 1998), an inside-out accretion model (Adams, Graff, \& Richstone 2001), and a self-interacting dark matter model (Ostriker 2000) or star formation-regulated model (Burkert \& Silk 2001). As an alternative model for $\mathrm{BH}$ growth, Umemura (2001) suggested a radiation-hydrodynamic model incorporating the physics of angular momentum transfer. The growth history of SMBHs is, however, rather uncertain. Two scenarios are involved in modeling these BHs: one is that SMBHs can grow out of low-mass "seed" BHs through accretion (Rees 1984), and another is that SMBHs grow by merging (Barkana, Haiman, \& Ostriker 2001).

It is widely believed that SMBHs were built up during the quasar epoch, around $z \sim 2-3$, where $z$ is the redshift of the quasars. However, observations of optically bright quasars show that if the quasars were continuously radiating, they would grow too large and run out of fuel (Haehnelt et al. 
1998; Richstone et al. 1998). On the other hand, if the quasar disk were unstable, then quasars would be intermittent and mostly nonradiating, with corresponding long periods of quiescence, resolving the old fueling problem in quasars (Shields \& Wheeler 1978). This implies that the growth of $\mathrm{BHs}$ through accreting gas happens only intermittently (Small \& Blandford 1992).

The S-shaped ionization instability first found to be responsible for the outbursts of cataclysmic variables (Meyer \& Meyer-Hoffmeister 1984; Smak 1984) also operates in binary systems containing accreting neutron stars or BHs. The time evolution of the limited cyclic behavior is caused by the thermal and viscous instability of the disk in the region of partial ionization of the hydrogen and leads to the transient behavior of many sources on a timescale of years (King 1995; Cannizzo 1993; Hameury et al. 1998). It is suggested that a similar instability may occur in a quasar disk (Lin \& Shields 1986; Cannizzo 1992; Mineshige \& Shields 1990; Clarke \& Shields 1989; Siemiginowska, Czerny, \& Kostyunin 1996; Hatziminaoglou, Siemiginowska, \& Elvis 2001), with the expected timescale being thousands to millions of years. Such an instability may modify the accretion rate in the central part of the flow and thus be responsible for a temporary change of the object status from highly active to quiescent on similar timescales. Burderi, King, \& Szuszkiewicz (1998) have summarized a decade of work on quasar disk instabilities and conclude that all observed AGNs are in the outburst phase. For a central BH with a mass of $10^{8} M_{\odot}$, the recurrence timescale of the Sshaped instability is about $10^{6} \mathrm{yr}$, of which the quasar disk spends about $10 \%$ of the time in outburst phase and $90 \%$ in the quiescent phase (Siemiginowska \& Elvis 1999). It is thus suggested that the bright quasars we observe today are in a highly active state, and the faint or dormant systems are simply those in quiescent states.

Motivated by the above investigations, we here develop an alternative accretion model associated with the S-shaped instability of the quasar disks for the growth of BHs harbored in quasar host galaxies. The mass relation between the $\mathrm{BH}$ and the galactic bulge in which the quasar lies is also discussed. Contrary to the former $\mathrm{BH}$ growth model through accretion (Rees 1984), we address the growth of the $\mathrm{BHs}$ using the ionization instability of the quasar disk. The behavior of the disk is successively "drought," "inundation," and "deluge" (Blandford 2002) in the lower, middle, and upper branches of the quasar disk, respectively. This paper is organized as follows: in $\S 2$ we discuss the accretion features of the quasar disk around a $\mathrm{BH}$ in different phases and study the growth of the $\mathrm{BH}$ with the evolution of the quasar disk, the BH-bulge mass relation is discussed in $\S 3$, and finally, we give our discussion and conclusion in $\S 4$.

\section{THE GROWTH OF BHs ASSOCIATED WITH THE S-SHAPED IONIZATION INSTABILITY OF QUASARS}

We first assume that there is a seed $\mathrm{BH}$ in the center of a quasar host galaxy with a mass much less than its presentday mass; for example, a seed $\mathrm{BH}$ could be formed from an immediate coalescence of the small-mass BHs contained in merging galaxies (Begelman, Blandford, \& Rees 1980; Cattaneo, Haehnelt, \& Rees 1999) or from an early-formed massive BH with $10^{6} M_{\odot}$ (Umemura, Loeb, \& Turner 1993). A massive $\mathrm{BH}$ with $\sim 10^{5} M_{\odot}$ can also be formed by the collapse of a rotating supermassive star (Baumgarte \& Shapiro
1999; Saijo et al. 2002; Carr, Bond, \& Arnett 1984; Nakamura \& Umemura 2001). The seed BH will grow as long as there is a gas supply for the inner disk. This gas reservoir is replenished by viscous inflow from the outer disk into the inner region of the disk and augmented by the increase in the inner radius due to the growth in the $\mathrm{BH}$ mass. A sufficient cold gas with a transfer rate $\dot{M}_{\text {ext }}$ could be supplied from a quasar host galaxy, for instance, mass loss from stars and stellar disruption and collisions, if the seed BH is embedded in densely populated stellar systems (Tsuruta 1977), and from progenitor galaxies if the seed BH was formed in major mergers (Kauffmann \& Haehnelt 2000). According to model (i) of Cattaneo et al. (1999), $\dot{M}_{\text {ext }}$ is proportional to the mass that has formed stars in the host galaxy. We take $\dot{M}_{\text {ext }}$ as a model parameter in this paper. If there is a lack of any limiting factor (Burkert \& Silk 2001), all the gas supply will eventually be accreted and fall into the $\mathrm{BH}$. Within the framework of our model, the Eddington limit (Small \& Blandford 1992) is used for the growth of the $\mathrm{BH}$, and the ionization instability of the quasar disk provides the self-regulation that limits the growth of the central $\mathrm{BH}$ in the three different phases.

\subsection{The S-shaped Ionization Instability of the Quasar Disk}

The most realistic scenario for an accretion disk around a $\mathrm{BH}$ is time dependent. The stationary local disk solutions form a characteristic S-shape on the $\dot{M}-\Sigma$ (accretion ratesurface density) plane. The accretion disk is thermally and viscously unstable when $\partial \dot{M} / \partial \Sigma<0$ (Meyer \& MeyerHoffmeister 1984). This is the case for accretion rates in the range $\left(\dot{M}_{1}, \dot{M}_{2}\right)$, where the S-shape has a negative slope (Fig. 1). A characteristic S-shaped instability consists of three branches (lower, middle, and upper branches), creating three possible accretion phases of the quasar disk. In the case of Keplerian motion, the surface density evolution can

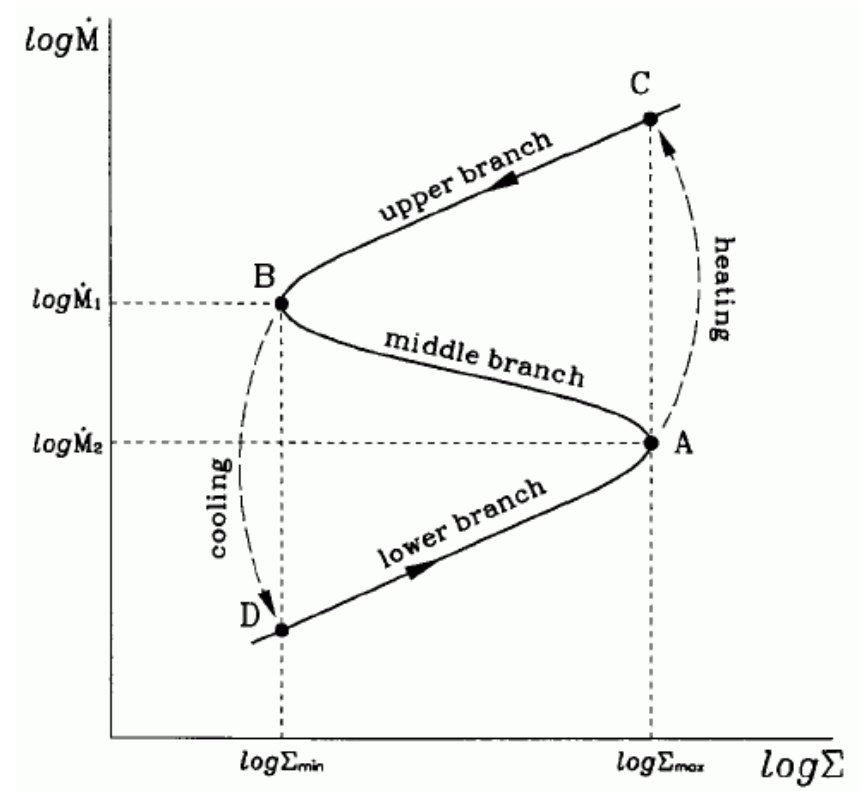

FIG. 1.-Relationship between local surface density and accretion rate $(\dot{M}-\Sigma)$ resulting from the instability of the quasar accretion disk (Lu, Cheng, \& Zhao 2000). 
be written (Pringle 1981; Smak 1984) as

$$
\begin{aligned}
\frac{\partial \Sigma}{\partial t}= & \frac{3}{r} \frac{\partial}{\partial r}\left[r^{1 / 2} \frac{\partial}{\partial r}\left(\nu \Sigma r^{1 / 2}\right)\right]+\frac{1}{2 \pi r} \frac{\partial \dot{M}}{\partial r} \\
& +\frac{1}{\pi r^{1 / 2}} \frac{\partial}{\partial r}\left[\left(r^{1 / 2}-r_{\text {out }}^{1 / 2}\right) \frac{\partial \dot{M}}{\partial r}\right],
\end{aligned}
$$

where $r_{\text {out }}$ is the outer radius of the disk and $\nu$ is the kinematic viscosity coefficient. The local accretion rate is given by

$$
\dot{M}=6 \pi r^{1 / 2} \frac{\partial}{\partial r}\left(r^{1 / 2} \nu \Sigma\right) .
$$

Equation (1) describes a disk that is continuously supplied not only with matter but also with angular momentum. If the mass inflow is variable with time, the thermally stable upper branch of the S-shaped instability ("active" phase) is characterized by an accretion rate higher than the stationary value, while the stable lower branch ("quiescence" phase) has a lower accretion rate than the stationary accretion rate, and so gas is accumulated in the outer region of the disk (Siemiginowska et al. 1996).

In this model, the disk is assumed to be in the configuration of an advection-dominated inflow-outflow solution (ADIOS; Blandford \& Begelman 1999) in the lower branch, in which most of the material transferred from the quasar host galaxy to the accretion disk is ejected in the form of an outflowing wind. This idea of an ADIOS configuration assumed in the lower branch is based on the arguments of Becker, Subramanian, \& Kazanas (2001). First, Narayan, McClintock, \& Yi (1996) and Narayan, Barret, \& McClintock (1997) have argued that the advectiondominated accretion flow (ADAF) model is far superior in explaining the broadband energy spectra of the lowluminosity (quiescent) state of $\mathrm{BH}$ binary systems than the standard optically thick and geometrically thin disk model (Shakura \& Sunyaev 1973); Ho (1996) subsequently applied the ADAF model to low-luminosity AGNs. Second, recent ADAF simulations of Igumenshchev \& Abramowicz (1999) indicated that bipolar outflows may be produced when the Shakura \& Sunyaev (1973) viscosity parameter $\alpha \sim 1$. Furthermore, if this is possible, then it appears that many underluminous $\mathrm{BH}$ systems such as $\mathrm{Sgr} \mathrm{A}^{*}$ may possess relativistic outflows (Falcke 1999; Donea, Falcke, \& Biermann 1999). Finally, Yuan et al. (2002) have recently also proposed that the combination of an ADAF configuration and outflows can explain the broadband energy spectra of several low-luminosity AGNs successfully. We therefore assume in this paper that the lower branch accretion disk is in the ADIOS configuration.

When the clumped mass in the outer region is transferred into the inner region by viscosity, the central seed $\mathrm{BH}$ gains mass and begins to grow. At the same time, when the surface density of the disk increases to a limit critical value $\Sigma_{A}$ (Fig. 1), the ionization instability occurs, and the disk jumps from the lower branch to the upper branch. In the upper branch, the gas in the disk is completely ionized, and the disk returns to stability. Unlike the lower branch, in the upper branch the disk is highly active, and radiation from the disk reaches the highest possible luminosity, restricted only by the Eddington limit. At this stage, the observed galaxy is naturally in a highly active phase, and the quasar is bright (Ho 1996). However, the upper branch may become radiation- pressure dominated and thus may be thermally unstable. The stabilizing mechanism in the upper branch may differ from that in the lower branch (Janiuk \& Czerny 2002). A hot outflowing wind in the upper branch with different properties from that in the lower branch may be produced, carrying both angular momentum and matter into the disk. The mass-loss rate of the outflowing wind in the upper branch is negligible compared to the accretion rate into the BH (Janiuk \& Czerny 2002). Energetically, the request for such an outflow in the upper branch is consistent with observations (Fender et al. 1999; Reeves et al. 2001).

After an outburst, the outer disk attempts to refill the central disk via steady inflow and begin the next cycle. The whole cycle repeats roughly on the viscous timescale of the outer disk. Because the recurrence timescale of the disk is longer, the individual quasar changes too slowly to be observed in the Galaxy. It is reasonable to assume that every quasar, as well as the growth of the $\mathrm{BH}$, has already experienced a sufficiently large number of cycles between the quiescent and active phase in the cosmic time.

\subsection{The Available Gas Mass Accumulated into the Quasar Disk}

In $\S 2.1$ we analyzed the evolution of the quasar disk along the S-shaped instability and how the BH gains its mass. It is interesting to calculate quantitatively the $\mathrm{BH}$ growth in the three phases in one cycle. We first estimate how much mass from the quasar host galaxy can be transferred and accumulated into the outer region of the disk. Considering our assumption that the disk in the lower branch is in the ADIOS configuration and that the mass loss of the wind from the quasar disk in the upper branch is negligible (Janiuk \& Czerny 2002), the transferred mass from the host galaxy to the disk can thus be divided into two components as the quasar disk evolves over one cycle: one component is the infalling material with mass $\left(\Delta M_{\mathrm{bh}}\right)_{\mathrm{acc}}$, which is also the available gas mass accumulated into the outer region of the quasar disk around the $\mathrm{BH}$; the other is the outflowing material with mass $\Delta M_{\text {out }}$, which is ejected into the interstellar medium in which the quasar lies. To calculate $\Delta M_{\text {out }}$ and $\left(\Delta M_{\mathrm{bh}}\right)_{\text {acc }}$, we use the pseudo-Newtonian relativistic ADIOS (RADIOS) model proposed by Becker et al. (2001) to describe the relation between the energy emitted into the wind and the amount of mass per unit radius per unit time, which is

$$
\begin{aligned}
\left(\frac{l_{\mathrm{bh}}}{\dot{m}}\right)_{\text {out }}= & \epsilon \int_{r_{\text {in }}}^{r_{\text {outer }}} \frac{r^{1 / 2}(r+6)}{(r-2)^{3}} d r \\
& \times\left[\int_{r_{\text {in }}}^{r_{\text {outer }}} \frac{r^{1 / 2}(r-6)}{(r-2)^{2}} d r\right]^{-1},
\end{aligned}
$$

where $\epsilon$ must satisfy $\max \left(\Omega_{0}^{2} / 4,\left(5 \Omega_{0}^{2}-1\right) / 4\right)<\epsilon<\Omega_{0}^{2}$, which ranges over $0.025<\epsilon<0.1, \Omega_{0}^{2}=0.1, \Omega_{0}$ is a positive constant, $r$ is the radius expressed in gravitational radii $\left(R_{g}=G M_{\mathrm{bh}} / c^{2}\right), l_{\mathrm{bh}}=L_{\mathrm{bh}} / L_{\mathrm{Edd}}$, and $\dot{m}=\dot{M}_{\text {out }} / \dot{M}_{\mathrm{Edd}}$. The quantity $L_{\mathrm{bh}}$ is the bolometric luminosity emitted by material accreting onto the $\mathrm{BH}$, and $\dot{M}_{\text {out }}$ is the mass-loss rate. The quantities $\dot{M}_{\text {Edd }}$ and $L_{\text {Edd }}$ are the Eddington accretion rate and luminosity, respectively, and they satisfy $L_{\text {Edd }}=0.1 \dot{M}_{\text {Edd }} c^{2}$. The quantity $r_{\text {in }}$ is the inner radius of the disk, and $r_{\text {outer }}$ is the outer radius at which the hot inner region transitions into the optically thick, cool outer disk. 
For this reference model, we adopt $r_{\text {in }}=6$ and $r_{\text {outer }} \sim 10^{2}$ (Narayan et al. 1996, 1997), which gives a numerical value for the integral in equation (3) of $\sim 0.03$.

In the case of the ADIOS situation, the accumulated mass in the outer region of the quasar disk and the outflowing mass on the viscous timescale $\left(t_{\mathrm{vis}}\right)$ in one cycle can be estimated as

$$
\begin{gathered}
\left(\Delta M_{\mathrm{bh}}\right)_{\mathrm{acc}} \sim\left(\dot{M}_{\mathrm{bh}}\right)_{\mathrm{acc}} t_{\mathrm{vis}}, \\
\Delta M_{\mathrm{out}} \sim \dot{M}_{\mathrm{out}} t_{\mathrm{vis}},
\end{gathered}
$$

where $\left(\dot{M}_{\mathrm{bh}}\right)_{\text {acc }}$ is the accumulated mass accretion rate in the quasar disk around the $\mathrm{BH}$. Eventually, the accumulated material on the quasar disk should fall into the $\mathrm{BH}$. We use the formula of $L_{\mathrm{bh}} \sim 0.1\left(\dot{M}_{\mathrm{bh}}\right)_{\mathrm{acc}} c^{2}$ to estimate $\left(\dot{M}_{\mathrm{bh}}\right)_{\mathrm{acc}}$ by taking the integrated fluences of the outbursts of each quasar source and assuming a radiative efficiency of 0.1 (which provides the total mass accreted in the outburst; Menou, Narayan, \& Lasota 1999). Combining equations (3) and (4), we obtain

$$
\left(\Delta M_{\mathrm{bh}}\right)_{\mathrm{acc}} \sim 0.03 \epsilon \dot{M}_{\mathrm{out}} t_{\mathrm{vis}} .
$$

Equation (6) gives the available maximum mass in the outer region of the disk in one cycle. Letting the values of $\epsilon$ vary as $0.025<\epsilon<0.1$, we get

$$
7.5 \times 10^{-4} \dot{M}_{\text {out }} t_{\text {vis }} \leq\left(\Delta M_{\text {bh }}\right)_{\text {acc }} \leq 3 \times 10^{-3} \dot{M}_{\text {out }} t_{\text {vis }} .
$$

We conclude that $\left(\Delta M_{\mathrm{bh}}\right)_{\mathrm{acc}}$ is about $10^{-3}$ of the total outflowing mass. Combining $\dot{M}_{\text {ext }} t_{\text {vis }}=\Delta M_{\text {out }}+\left(\Delta M_{\text {bh }}\right)_{\text {acc }}$ and equation (5), we thus have

$$
\Delta M_{\text {out }} \sim \dot{M}_{\text {ext }} t_{\mathrm{vis}}
$$

and then

$$
\left(\dot{M}_{\mathrm{bh}}\right)_{\mathrm{acc}} \sim 0.03 \epsilon \dot{M}_{\mathrm{ext}} .
$$

We treat $\dot{M}_{\text {ext }}$ as a model parameter, which is determined by the total mass of stars formed in the host galaxy (Cattaneo et al. 1999).

\subsection{The Mass Growth of the BH along the S-shaped Instability in One Cycle}

In this section we study the growth of the central BH along the S-shaped instability in one cycle. We argue that in order to realize the next cycle of the S-shaped instability, the accumulated mass $\left(\Delta M_{\mathrm{bh}}\right)_{\mathrm{acc}}$ of the quasar disk must be either swallowed by the $\mathrm{BH}$ or ejected away in the upper branch when the quasar is in the highly active state. Since the upper branch of the S-shaped instability involves physics that are currently open-ended (Janiuk \& Czerny 2002; Blandford 2002; Mirabel \& Rodríguez 1999), we estimate the properties of the outflowing and infalling mass in the upper branch according to observations. It is known that jets are among the most ubiquitous of astrophysical phenomena and are associated with objects ranging from protostars and binary systems in our Galaxy to SMBHs in the centers of AGNs. Large asymptotic Lorentz factors are implied by observations of superluminal motion in many blazars (Dermer \& Schlickeiser 1992; Vermeulen \& Cohen 1994), and the EGRET instrument on board the Compton Gamma Ray Observatory has also detected intense $\gamma$-ray flares from many of these sources (Wehrle et al. 1998;
Kataoka et al. 1999). The mechanisms responsible for producing the observed jets are still poorly understood, but collimated outflows in general appear to be associated with objects that derive their luminosity from the accretion of matter onto a gravitating object (Dermer \& Schlickeiser 1992). The observations of relativistic jets show that asymptotic Lorentz factors are about $\gamma_{\mathrm{jet}} \geq 10$ (Mészáros \& Rees 1992; Quinn 1996). For an accretion rate $\dot{M}_{\text {bh }}$, the emitted luminosity can be estimated as

$$
L_{\mathrm{bh}}=0.1 \dot{M}_{\mathrm{bh}} c^{2} .
$$

If the outflow gas expands adiabatically, the total energy per unit mass in the jet at a rate $\dot{M}_{\text {jet }}$ follows

$$
L_{\mathrm{jet}}=\gamma_{\mathrm{jet}} \dot{M}_{\mathrm{jet}} c^{2} .
$$

It therefore follows immediately that

$$
\frac{\dot{M}_{\text {jet }}}{\dot{M}_{\text {bh }}}=\frac{1}{10 \gamma_{\text {jet }}} \ll 1 \text {. }
$$

Equation (11) shows that the mass flux in the jet in the upper branch is less than only $1 \%$ of the mass flux into the $\mathrm{BH}$. This simple energetic argument predicts that mass carried out by the outflow jet can be ignored when compared to the mass accreted into the $\mathrm{BH}$ in the upper branch. We therefore neglect the mass loss in the upper branch. This means that all accumulated mass onto the quasar disk will be swallowed by the $\mathrm{BH}$ before a new cycle starts. Consequently, we have

$$
\Delta M_{\mathrm{bh}}=\left(\Delta M_{\mathrm{bh}}\right)_{\mathrm{acc}}=0.03 \epsilon \dot{M}_{\mathrm{ext}} t_{\mathrm{vis}},
$$

where $\Delta M_{\mathrm{bh}}$ is the total mass growth of the $\mathrm{BH}$ gained in each cycle of the S-shaped instability.

We are also interested in the details, with observation constraints, of how the BH grows up along the S-shaped instability of the quasar disk. It is known that the surface density and the local accretion rate of the quasar disk evolve on the viscous timescale $t_{\mathrm{vis}} \sim R / v_{R}$ (Lightman 1974; where $v_{R}$ is the radial velocity and $R$ is the accretion radius of the disk); thus, the lifetime of a quasar accretion disk in each cycle is $t_{\mathrm{vis}}$. The recurrence timescale $t_{\text {current }}$ of the S-shaped instability of the quasar disk is addressed as $t_{\text {current }} \sim t_{\text {vis }}$, which operates over $10^{4}-10^{6}$ yr (Siemiginowska et al. 1996). Timescales spent by the quasar disk in the lower, transition, and upper branches in one cycle are $t_{\text {low }}, t_{\text {tran }}$, and $t_{\text {up }}$, respectively. Then, the timescales spent by the quasar disk in the three different phases are (Siemiginowska \& Elvis 1999)

$$
\begin{aligned}
t_{\text {low }} & \sim 0.75 t_{\text {vis }}, \\
t_{\text {up }} & \sim 00.1 t_{\text {vis }}, \\
t_{\text {tran }} & \sim 0.15 t_{\text {vis }} .
\end{aligned}
$$

We assume that the total growth mass $\Delta M_{\mathrm{bh}}$ of the $\mathrm{BH}$ in one cycle is built up when the quasar disk passes the three different phases. Then, the total growth mass of the $\mathrm{BH}$ in one cycle is

$$
\Delta M_{\mathrm{bh}}=\dot{M}_{\mathrm{bhl}} t_{\mathrm{low}}+\dot{M}_{\mathrm{bhu}} t_{\mathrm{up}}+\dot{M}_{\mathrm{bht}} t_{\mathrm{tran}},
$$

where $\dot{M}_{\text {bhl }}, \dot{M}_{\text {bht }}$, and $\dot{M}_{\text {bhu }}$ are the accretion rates of the $\mathrm{BH}$ in the lower, transition, and upper branches, 
respectively. Each accretion rate is limited by the Eddington accretion rate.

The accretion rate in the transition phase can be estimated from the features of the S-shaped instability. It is noted that the ionization instability of the quasar disk occurs when the accretion rates of the disk are in the range $\left(\dot{M}_{2}, \dot{M}_{1}\right)$ (see Fig. 1). We thus approximate

$$
\dot{M}_{\text {bht }}=\frac{1}{2}\left(\dot{M}_{\mathrm{bhl}}+\dot{M}_{\mathrm{bhu}}\right) \text {. }
$$

Inserting equations (13)-(15) and (17) into equation (16), we get

$$
\begin{aligned}
\Delta M_{\mathrm{bh}} \approx & 0.75 \dot{M}_{\mathrm{bhl}} t_{\mathrm{vis}}+0.1 \dot{M}_{\mathrm{bhu}} t_{\mathrm{vis}} \\
& +0.075\left(\dot{M}_{\mathrm{bhl}}+\dot{M}_{\mathrm{bhu}}\right) t_{\mathrm{vis}} .
\end{aligned}
$$

Comparing equation (12) with equation (18), we get

$$
0.03 \epsilon \dot{M}_{\mathrm{ext}}=0.825 \dot{M}_{\mathrm{bhl}}+0.175 \dot{M}_{\mathrm{bhu}} .
$$

If $0.01 \leq \dot{M}_{\mathrm{bhl}} / \dot{M}_{\mathrm{bhu}} \leq 0.1$ (Mirabel \& Rodríguez 1999), the accretion rates in the three phases can be estimated roughly as

$$
\begin{aligned}
& 1.64 \times 10^{-3} \epsilon \dot{M}_{\mathrm{ext}} \leq \dot{M}_{\mathrm{bhl}} \leq 1.16 \times 10^{-2} \epsilon \dot{M}_{\mathrm{ext}}, \\
& 1.16 \times 10^{-1} \epsilon \dot{M}_{\mathrm{ext}} \leq \dot{M}_{\mathrm{bhu}} \leq 1.64 \times 10^{-1} \epsilon \dot{M}_{\mathrm{ext}}, \\
& 2.28 \times 10^{-2} \epsilon \dot{M}_{\mathrm{ext}} \leq \dot{M}_{\mathrm{bht}} \leq 6.38 \times 10^{-2} \epsilon \dot{M}_{\mathrm{ext}}
\end{aligned}
$$

If $\dot{M}_{\text {bhu }}=\dot{M}_{\text {Edd }}$, the limits of $\dot{M}_{\text {ext }}$ required by our model can be inferred from equation (20), which gives

$$
6 M_{\odot} \mathrm{yr}^{-1} \leq \dot{M}_{\text {ext }} \leq 86 M_{\odot} \mathrm{yr}^{-1}
$$

for $\epsilon=0.1$ and

$$
24.4 M_{\odot} \mathrm{yr}^{-1} \leq \dot{M}_{\mathrm{ext}} \leq 345 M_{\odot} \mathrm{yr}^{-1}
$$

for $\epsilon=0.025$.

The mass growth of the $\mathrm{BH}$ in the three phases of one cycle can be obtained by

$$
\begin{aligned}
& 1.23 \times 10^{-3} \epsilon \dot{M}_{\text {ext }} t_{\text {vis }} \leq \Delta M_{\text {bhl }} \leq 8.7 \times 10^{-3} \epsilon \dot{M}_{\text {ext }} t_{\text {vis }} \\
& 1.16 \times 10^{-2} \epsilon \dot{M}_{\text {ext }} t_{\text {vis }} \leq \Delta M_{\text {bhu }} \leq 1.64 \times 10^{-2} \epsilon \dot{M}_{\text {ext }} t_{\text {vis }} \\
& 3.42 \times 10^{-3} \epsilon \dot{M}_{\text {ext }} t_{\text {vis }} \leq \Delta M_{\text {bht }} \leq 9.57 \times 10^{-3} \epsilon \dot{M}_{\text {ext }} t_{\text {vis }}
\end{aligned}
$$

where $\Delta M_{\mathrm{bhl}}=\dot{M}_{\mathrm{bhl}} t_{\mathrm{low}}, \Delta M_{\mathrm{bhu}}=\dot{M}_{\mathrm{bhu}} t_{\mathrm{up}}$, and $\Delta M_{\mathrm{bht}}=$ $\dot{M}_{\text {bht }} t_{\text {tran }}$ are the mass growth of the BH in the lower, upper, and transition branches, respectively.

\section{THE PRESENT MASS OF THE BH AND THE BH-BULGE MASS RELATION}

The total mass of the resulting $\mathrm{BH}$ at present is the sum of the growth mass through the seed $\mathrm{BH}$ accreting in each cycle during the Hubble time. Taking into account the model assumption that the accretion rate onto the quasar disk is limited by Eddington limits, we can rewrite equation (12) as

$$
\frac{d M_{\mathrm{bh}}}{d t}= \begin{cases}0.03 \epsilon \dot{M}_{\mathrm{ext}}, & 0.03 \epsilon \dot{M}_{\mathrm{ext}} \leq \dot{M}_{\mathrm{Edd}}, \\ \dot{M}_{\mathrm{Edd}}, & 0.03 \epsilon \dot{M}_{\mathrm{ext}} \geq \dot{M}_{\mathrm{Edd}}\end{cases}
$$

The solutions of equation (24) are as follows:
1. If $0.03 \epsilon \dot{M}_{\mathrm{ext}} \leq \dot{M}_{\mathrm{Edd}}$,

$$
M_{\mathrm{bh}}(t)=0.03 \epsilon \dot{M}_{\mathrm{ext}} t+M_{\mathrm{bh}}(0),
$$

where $t$ ranges over $0 \leq t \leq t_{\mathrm{H}}$ and $t_{\mathrm{H}}$ is the Hubble timescale $\left(t_{\mathrm{H}}=10 \mathrm{Gyr}\right)$. The quantities $M_{\mathrm{bh}}(t)$ and $M_{\mathrm{bh}}(0)$ are the present $\mathrm{BH}$ mass and the seed $\mathrm{BH}$ mass, respectively.

For $0.025<\epsilon<0.1$, the seed $\mathrm{BH}$ mass $M_{\mathrm{bh}}(0)$ is

$$
2 \times 10^{6} M_{\odot} \leq M_{\mathrm{bh}}(0) \leq 3 \times 10^{7} M_{\odot} .
$$

Then, the present $\mathrm{BH}$ mass becomes $2 \times 10^{8}$ and $3 \times 10^{9}$ $M_{\odot}$ for seed $\mathrm{BH}$ masses of $2 \times 10^{6}$ and $3 \times 10^{7} M_{\odot}$, respectively.

2. If $0.03 \epsilon \dot{M}_{\mathrm{ext}} \geq \dot{M}_{\mathrm{Edd}}$,

$$
M_{\mathrm{bh}}(t)=M_{\mathrm{bh}}(0) e^{\left(t / t_{\mathrm{Edd}}\right)},
$$

where $t_{\text {Edd }}$ is the Eddington accretion time. This solution is not important, because the BH will first quickly grow exponentially and then switch back to the first solution (eq. [25]). Since the time spent in exponential growth is much less than the Hubble time, this stage can be ignored unless the seed $\mathrm{BH}$ mass is extremely small. Consequently, the solutions of equation (24) suggest that a typical quasar $\mathrm{BH}$ with a final mass $2 \times 10^{8} M_{\odot}$ can be grown from a seed BH with mass $2 \times 10^{6} M_{\odot}$.

Assuming that the material transferred from the host galaxy is proportional to the mass that has formed stars, this situation is strictly true in model (i) of Cattaneo et al. (1999), and $\Delta M_{\text {out }} \approx \dot{M}_{\text {ext }} t_{\text {vis }} \approx M_{\text {bulge, }}$, where $M_{\text {bulge }}$ is the mass of the host galactic bulge. Taking into account equations (7) and (12) and letting $0.025 \leq \epsilon \leq 0.1$, we thus obtain the mass ratio of the $\mathrm{BH}$ to the galactic bulge:

$$
7.5 \times 10^{-4} \leq \frac{M_{\mathrm{bh}}}{M_{\text {bulge }}} \leq 3 \times 10^{-3} .
$$

\section{DISCUSSION AND CONCLUSION}

A quasar disk model associated with the ionization instability is proposed to account for the buildup of SMBHs in quasar host galaxies. The growth of the $\mathrm{BH}$ is studied when the quasar disk evolves along an S-shaped instability. We show that the main growth of the $\mathrm{BH}$ occurs in the upper branch, when the quasar disk is in a highly active state. Two model parameters $\epsilon$ and $\dot{M}_{\text {ext }}$ are involved. Although in quasar accretion disks the gas supply mechanism is unknown (Siemiginowska et al. 1996), the limits for an available gas supply with $6 M_{\odot} \mathrm{yr}^{-1} \leq \dot{M}_{\mathrm{ext}} \leq 86 M_{\odot} \mathrm{yr}^{-1}$ from the quasar host galaxy are deduced from observations and the limits of the Eddington accretion rate when the disk evolves in the upper branch. In fact, there is no physical reason to limit the mass transfer rate to the Eddington rate. However, if the radiation pressure generated within an object exceeds the gravitational force, then the outer layer of the object can be blown away (Siemiginowska \& Elvis 1999). Therefore, we assume that during the disk outburst radiation from the disk is restricted by the Eddington limit, which is defined by the balance between the total gravitational force and radiation pressure. This assumption nevertheless does not affect our conclusions significantly, because a super-Eddington accretion will become sub-Eddington as the $\mathrm{BH}$ mass increases. 
It has been pointed out by Menou \& Quataert (2001) that the ionization instability may not be able to produce the observed large-amplitude luminosity transitions between the low and high states in AGNs. However, their argument was based on the assumption that the accretion disks in both the low and high states are in the standard optically thick and geometrically thin configuration, which is quite different from the basic assumption we made in this paper. It is unclear if the point raised by Menou \& Quataert (2001) is also valid here.

Our conclusion can be summarized as follows: (1) We first demonstrate that the BHs of the quasar host galaxies can grow up through accretion with two model parameters. With $0.025<\epsilon<0.1$, a BH with mass $2 \times 10^{8} M_{\odot}$ can grow up from a seed BH with mass $2 \times 10^{6} M_{\odot}$. (2) With the assumption of an ADIOS model in the lower branch, the observed BH-bulge mass relation (Magorrian et al. 1998) can be inferred, which is $7.5 \times 10^{-4} \leq M_{\mathrm{bh}} / M_{\text {bulge }} \leq$
$3 \times 10^{-3}$ in our model parameters. (3) Furthermore, in the present scheme, a massive seed $\mathrm{BH}$ is required in order to build up an SMBH through accretion if the gas is provided from the quasar host galaxy. This hints that a low-mass stellar BH may not grow to an SMBH unless there is no Eddington limit. The details for the formation of a massive seed $\mathrm{BH}\left(\sim 10^{6} M_{\odot}\right)$ and whether there is a sufficient cold gas supplied to the $\mathrm{BH}$ are beyond the scope of this paper.

We thank the anonymous referee for insightful and constructive comments and suggestions, which have improved the manuscript significantly. We are grateful to Y. F. Huang and X. Z. Zheng for helpful discussions. This work was supported by an RGC grant of the Hong Kong Special Administrative Region government, the National Natural Science Foundation of China under grant 10273011, the National 973 Project (NKBRSF G19990754), and the Special Funds for Major State Basic Research Projects.

\author{
Adams, F. C., Graff, D. S., \& Richstone, D. O. 2001, ApJ, 551, L31 \\ Barkana, R., Haiman, Z., \& Ostriker, J. P. 2001, ApJ, 558, 482 \\ Baumgarte, T. W., \& Shapiro, S. L. 1999, ApJ, 526, 941 \\ Becker, P., Subramanian, P., \& Kazanas, D. 2001, ApJ, 552, 209 \\ Begelman, M. C., Blandford, R. D., \& Rees, M. J. 1980, Nature, 287, 307 \\ Blandford, E. G., \& Begelman, M. C. 1999, MNRAS, 303, L1 \\ Blandford, R. D. 2002, in Proc. 2nd KIAS Astrophys. Workshop, \\ Current High-Energy Emission around Black Holes, ed. C.-H. Lee \& \\ H.-Y. Chang (Singapore: World Scientific), 199 \\ Boyle, B. J., \& Terlevich, R. J. 1998, MNRAS, 293, L49 \\ Burderi, L., King, A. R., \& Szuszkiewicz, E. 1998, ApJ, 509, 85 \\ Burkert, A., \& Silk, J. 2001, ApJ, 554, L151 \\ Cannizzo, C. J. 1992, ApJ, 385, 94 \\ 1993, ApJ, 419, 318 \\ Carr, B. J., Bond, J. R., \& Arnett, W. D. 1984, ApJ, 277, 445 \\ Cattaneo, A., Haehnelt, M., \& Rees, M. J. 1999, MNRAS, 308, 77 \\ Clarke, C. J., \& Shields, G. A. 1989, ApJ, 338, 32 \\ Dermer, C. D., \& Schlickeiser, R. 1992, Science, 257, 1642 \\ Donea, A. C., Falcke, H., \& Biermann, P. L. 1999, in ASP Conf. Ser. \\ 186, The Central Parsecs of the Galaxy, ed. H. Falcke, A. Cotera, \\ W. J. Duschl, F. Melia, \& M. J. Rieke (San Francisco: ASP), 162 \\ Fabian, A. C. 1999, MNRAS, 308, L39 \\ Falcke, H. 1999, in ASP Conf. Ser 186, The Central Parsecs of the Galaxy, \\ ed. H. Falcke, A. Cotera, W. J. Duschl, F. Melia, \& M. J. Rieke (San \\ Francisco: ASP), 148 \\ Fender, R., Garrington, S. T., McKay, D. J., Muxlow, T. W. B., Pooley, \\ G. G., Spencer, R. E., Stirlinge, A. M., \& Waltman, E. B. 1999, MNRAS, \\ 304,865 \\ Ferrarese, L., \& Merritt, D. 2000, ApJ, 539, L9 \\ Ferrarese, L., et al. 2001, ApJ, 555, L79 \\ Gebhardt, K., et al. 2000a, ApJ, 539, L13 \\ 2000b, ApJ, 543, L5 \\ Granato, G. L., Silva, L., Monaco, P., Panuzzo, P., Salucci, P., De Zotti, \\ G., \& Danese, L. 2001, MNRAS, 324, 757 \\ Haehnelt, M. G., \& Kauffmann, G. 2000, MNRAS, 318, L35 \\ Haehnelt, M. G., Natarajian, P., \& Rees, M. 1998, MNRAS, 300, 817 \\ Hameury, J.-M., Menou, K., Dubus, G., Lasota, J. R., \& Hure, J. M. 1998, \\ MNRAS, 298, 1048 \\ Hatziminaoglou, E. Siemiginowska, A., \& Elvis, M. 2001, ApJ, 547, 90 \\ Ho, L. C. 1996, in ASP Conf. Ser. 103, The Physics of LINERs in View of \\ Recent Observations, ed. M. Eracleous, A. Koratkar, C. Leitherer, \& \\ L. Ho (San Francisco: ASP), 103 \\ Igumenshchev, I. V., \& Abramowicz, M. A. 1999, MNRAS, 303, 309 \\ Janiuk, A., \& Czerny, B. 2002, ApJ, 576, 908 \\ Kataoka, J., et al. 1999, ApJ, 514, 138 \\ Kauffmann, G., \& Haehnelt, M. 2000, MNRAS, 311, 576
}

\section{REFERENCES}

King, A. R. 1995, X-Ray Binaries, ed. W. H. G. Lewin, J. van Paradijs, \& E. P. J. van den Heuvel (Cambridge: Cambridge Univ. Press), 419

Kormendy, J., \& Richstone, D. 1995, ARA\&A, 33, 581

Laor, A. 1998, ApJ, 505, L83

Lightman, A. P. 1974, ApJ, 194, 419

Lin, D. N. C., \& Shields, G. A. 1986, ApJ, 305, 28

Lu, Y., Cheng, K. S., \& Zhao, G. 2000, PASJ, 52, 711

Lynden-Bell, D. 1969, Nature, 223, 690

Magorrian, J., et al. 1998, AJ, 115, 2285

Menou, K., Narayan, R., \& Lasota, J. P. 1999, ApJ, 513, 811

Menou, K., \& Quataert, E. 2001, ApJ, 552, 204

Merritt, D., \& Ferrarese, L. 2001, MNRAS, 320, L30

Mészáros, P., \& Rees, M. J. 1992, MNRAS, 257, 29

Meyer, F., \& Meyer-Hoffmeister, E. 1984, A\&A, 132, 143

Mineshige, S., \& Shields, G. A. 1990, ApJ, 351, 47

Mirabel, I. F., \& Rodríguez, L. F. 1999, ARA\&A, 37, 409

Nakamura, F., \& Umemura, M. 2001, ApJ, 548, 19

Narayan, R., Barret, D., \& McClintock, J. E. 1997, ApJ, 482, 448

Narayan, R., McClintock, J. E., \& Yi, I. 1996, ApJ, 457, 821

Nelson, R. P., et al. 2000, MNRAS, 318, 18

Ostriker, J. P. 2000, Phys. Rev. Lett., 84, 5258

Page, M. J., Stevens, J. A., Mittaz, J. P. D., \& Carrera, F. J. 2001, Science, 294, 2516

Pringle, J. E. 1981, ARA\&A, 19, 137

Quinn, J. 1996, ApJ, 456, L83

Rees, M. J. 1984, ARA\&A, 22, 471

Reeves, J. N., et al. 2001, A\&A, 365, L116

Richstone, D., et al. 1998, Nature Suppl., 395, 14

Saijo, M., Baumgarte, T. W., Shapiro, S. L., \& Shibata, M. 2002, ApJ, 569, 349

Sanders, D. B., \& Mirabel, I. F. 1996, ARA\&A, 34, 749

Shakura, N. I., \& Sunyaev, R. A. 1973, A\&A. 24, 337

Shields, G. A., \& Wheeler, J. C. 1978, ApJ, 222, 667

Siemiginowska, A., Czerny, B., \& Kostyunin, V. 1996, ApJ, 458, 491

Siemiginowska, A., \& Elvis, M. 1999, Nature, 397, 476

Silk, J. \& Rees, M. J. 1998, A\&A, 331, L1

Smak, J. 1984, PASP, 96, 5

Small, T., \& Blandford, R. 1992, MNRAS, 259, 725

Tsuruta, S. 1977, A\&A, 61, 647

Umemura, M. 2001, ApJ, 560, L29

Umemura, M., Loeb, A., \& Turner, E. L. 1993, ApJ, 419, 459

van der Marel, R. P. 1999, AJ, 117, 744

Vermeulen, R. C., \& Cohen, M. H. 1994, ApJ, 430, 467

Wehrle, A. E., et al. 1998, ApJ, 497, 178

Yuan, F., Markoff, S., Falcke, F., \& Biermann, P. L. 2002, A\&A, 391, 139 